\title{
BMJ Open Early detection and management of major non-communicable diseases in urban primary healthcare facilities in Ethiopia: a study protocol for a type-3 hybrid implementation-effectiveness design
}

To cite: Yifter H, Omer A, Gugsa S, et al. Early detection and management of major non-communicable diseases in urban primary healthcare facilities in Ethiopia: a study protocol for a type-3 hybrid implementationeffectiveness design. BMJ Open 2021;11:e040564. doi:10.1136/ bmjopen-2020-040564

- Prepublication history and additional materials for this paper is available online. To view these files, please visit the journal online (http://dx.doi. org/10.1136/bmjopen-2020040564).

Received 16 May 2020 Revised 28 November 2020 Accepted 11 December 2020

Check for updates

(c) Author(s) (or their employer(s)) 2021. Re-use permitted under CC BY-NC. No commercial re-use. See rights and permissions. Published by BMJ.

For numbered affiliations see end of article.

Correspondence to

Afrah Omer;

afrahsanni@gmail.com

\section{ABSTRACT}

Introduction Integrating early detection and management of non-communicable diseases in primary healthcare has an unprecedented role in making healthcare more accessible particularly in low- and middle-income countries such as Ethiopia. This study aims to design, implement and evaluate an evidencebased intervention guided by the HEARTS technical package and implementation guide to address barriers and facilitators of integrating early detection and management of hypertension, diabetes mellitus and cardiovascular diseases in primary healthcare settings of Addis Ababa.

Methodology We will employ a type-3 hybrid implementation-effectiveness study from November 2020 to May 2022. This study will target patients $\geq 40$ years of age. Ten health centres will be randomly selected from each subcity of Addis Ababa. The study will have four phases: (1) Baseline situational analysis (PEN facility-capacity assessment, 150 observations of patient healthcare provider interactions and 697 patient medical record reviews), (2) Consolidated Framework for Implementation Research (CFIR) inspired qualitative assessment of barriers and facilitators (20 in-depth interviews of key stakeholders), (3) Design of intervention protocol. The intervention will have capacity enhancement components including training of noncommunicabledisease (NCDservice providers, provision of essential equipment/supporting materials and monthly monitoring and feedback and (4) Implementation monitoring and evaluation phase using the RE-AIM (reach, efficacy, adoption, implementation and maintenance) framework. Outcomes on early detection and management of NCDs will be assessed to examine the effectiveness of the study.

Ethics and dissemination plan Ethical clearance was obtained from the Addis Ababa University, College of Health Sciences Institutional Review Board and Addis Ababa Health Bureau. We plan to present the findings from this research in conferences and publish them in peerreviewed journals.
Strengths and limitations of this study

The study uses an implementation research framework to design interventions, analyse and report the findings of the study.

- The study uses a comprehensive baseline assessment of health facilities to inform the design of the intervention.

- The study will adapt the WHO HEARTS technical package and implementation guide to the local context to implement the intervention.

- The uncontrolled nature of the design might reduce the rigorousness of the study.

- As the outcome indicators for this study relies on routine medical records and observations, missing and observation bias might arise.

\section{INTRODUCTION}

Non-communicable diseases (NCDs) are the leading causes of death worldwide. Integrating cost-effective NCD interventions in primary healthcare, as the first point of care, has a great potential to reverse progression of disease, prevent complications, reduce hospitalisations, healthcare costs and out-ofpocket expenditures ${ }^{1}$ and make healthcare accessible. ${ }^{2}$ However, many African countries have health systems that do not meet the requirements for chronic disease care and are historically shaped around acute care. $^{3}$ Management of NCDs is challenging especially in low- and middle-income countries due to the double burden of communicable and non-communicable diseases, lack of human and material resources and other competing economic priorities in the countries. ${ }^{45}$ The long-term nature of many NCDs demand an integrated and comprehensive 
health system response ${ }^{6}$ and need to be sustained for a significant period of time. ${ }^{7}$

A total of 57 million deaths occurred globally in 2016; of which, 41 million $(71 \%)$ were due to NCDs, principally cardiovascular diseases (CVD), diabetes, cancer and chronic respiratory diseases. ${ }^{89}$ NCDs are responsible for 15 million premature adult deaths (occurring in those aged 30 to 69 years). Nearly $80 \%$ of these premature deaths caused by NCDs occurred in low- and middle-income countries. ${ }^{10}$ Though communicable diseases are still the leading cause of mortality in Africa, the burden of NCDs is rising rapidly and is projected to cause almost threequarters as many deaths as communicable, maternal, perinatal and nutritional diseases by 2020 and to exceed them as the most common causes of death by $2030 .{ }^{11}$ Similarly, in Ethiopia, the burden of NCDs is increasing at an alarming rate. According to WHO country profiles, currently, NCDs are responsible for almost 39\% of all deaths in Ethiopia. ${ }^{9}$

Strengthening the national capacity to integrate and scale-up care for major NCDs such as diabetes, CVD and respiratory disease in primary healthcare in low-resource settings were among the top recommendations. ${ }^{45}$ Over the years, the WHO has developed evidence informed guidelines to enhance the prevention, diagnosis and management of NCDs such as diabetes, CVD and respiratory disease at primary healthcare settings. Among these guidelines are the package of essential noncommunicable disease (PEN) and the total risk approach where cost-effective interventions for early detection of NCDs, non-pharmacological and pharmacological approaches for modification of risk factors and affordable medications for treatment of NCDs. ${ }^{4}$ In addition, in 2016, the HEARTS technical package was developed as a measure to avert CVD by addressing major modifiable risk factors including tobacco use, hypertension, high blood cholesterol and diabetes. ${ }^{5}$ The package includes simple and affordable tools (eg, clinical measurements, simple laboratory testing, CVD risk assessment charts and blood pressure measurement devices) for early detection and treatment of CVDs. Moreover, these two guidelines are aligned and complementary to each other. ${ }^{5}$

Studies in sub-Saharan Africa indicated the importance of NCD integration models ${ }^{12}$ and effectiveness of advice on diet and lifestyle modification to facilitate management of NCDs in resource poor settings. ${ }^{13}$ The government of Ethiopia also produced a national guideline, championing integration of early detection and management of NCDs in primary healthcare to address the national burden of NCDs. ${ }^{14}$

Despite these initiatives, there is lack of evidence on practical implementation of guidelines, as well as little research on barriers and facilitators of early detection and management of NCDs in Ethiopia.

This study aims to design, implement and evaluate an evidence-based intervention guided by the HEARTS technical package and implementation guide to address barriers and facilitators of integrating early detection and management of hypertension, diabetes mellitus and CVDs in primary healthcare settings of Addis Ababa. The intervention will comprise capacity enhancement components including development of training module and jobaids, training of NCD service providers, provision and/ or maintenance of essential equipment, guidelines and supporting materials and regular monitoring and feedback. With this study protocol, we will present how we will inform the design of the intervention and how we plan to implement and evaluate the intervention.

\section{METHODS AND MATERIALS \\ Study settings}

The study will be conducted in Addis Ababa, Ethiopia. Addis Ababa city administration is the capital city of the country. It is organised in 10 subcities, which are the second upper administration units next to the city administration. Addis Ababa has an estimated total population of 5.65 million, with an average density of 5645.61 people per square $\mathrm{km}$. According to the 2017/2018 Health and Health Related Indicators of Ethiopia, the city has 25 hospitals and 97 health centres. ${ }^{15} \mathrm{~A}$ health centre is the health service nearest to the public, where people receive direct and primary healthcare services. In Addis Ababa, a health centre serves approximately 25000 people under its catchment area. It provides both preventive and curative services and serves as a referral centre. ${ }^{16}$ Health centres are also the first contact points for NCD screening, detection and management. On average, health centres are staffed with 20 healthcare providers, of whom 5 healthcare providers work in adult outpatient departments (OPDs). ${ }^{16}$ Health centres report to subcity health bureaus, while the subcity health bureau is in charge of implementing a monthly mentorship programme as a standard procedure for each health centre providing NCD services in Addis Ababa. Mentors from subcities visit their respective health centres to supervise the quality of NCD services and provide written feedback to service providers and health centres.

Health centres in Addis Ababa are stratified in to 10 subcities. We excluded 27 health centres with an ongoing intervention on NCDs. From the remaining pool of 70 health centres, we randomly selected 1 health centre from each subcity to obtain a total of 10 health centres. Thus, the study will be conducted in 10 health centres (figure 1).

\section{Study design and period}

This study will employ a type-3 hybrid implementationeffectiveness design ${ }^{17}$ and will be conducted from November 2020 to May 2022. The study will be conducted in four phases, each described in detail below (figure 2):

Phase one: baseline situational analysis

This first phase will form the baseline assessment stage of the project and employ the following approaches. 


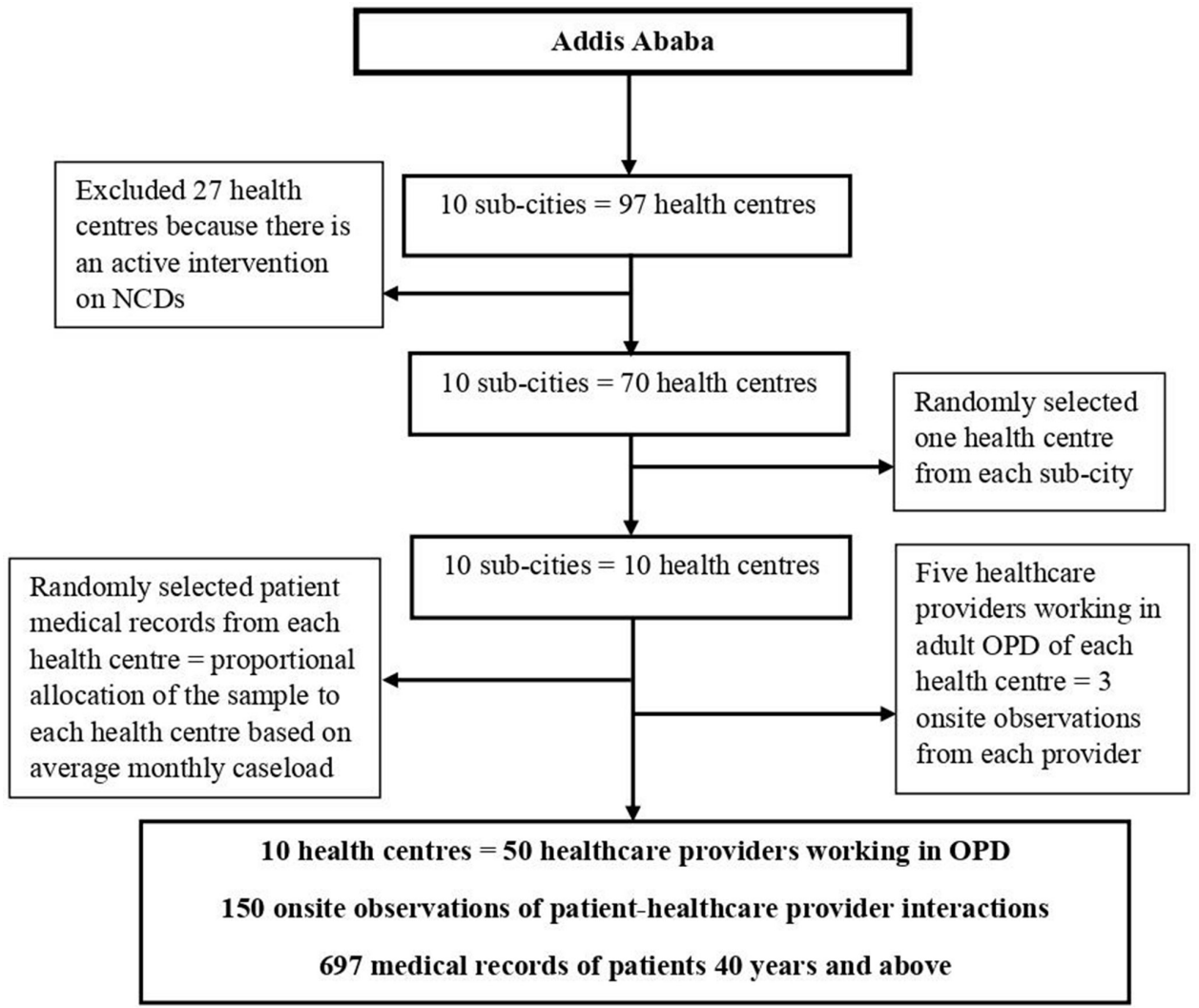

Figure 1 Sampling procedure and allocation of health centres, healthcare providers and patient medical records. NCDs, noncommunicable diseases; OPD, outpatient department.

1. Caseload of major NCDs including hypertension and diabetes in the selected 10 health centres during the previous 3 months before the baseline data collection will be summarised by reviewing the Health Management Information System (HMIS) reports of health centres.

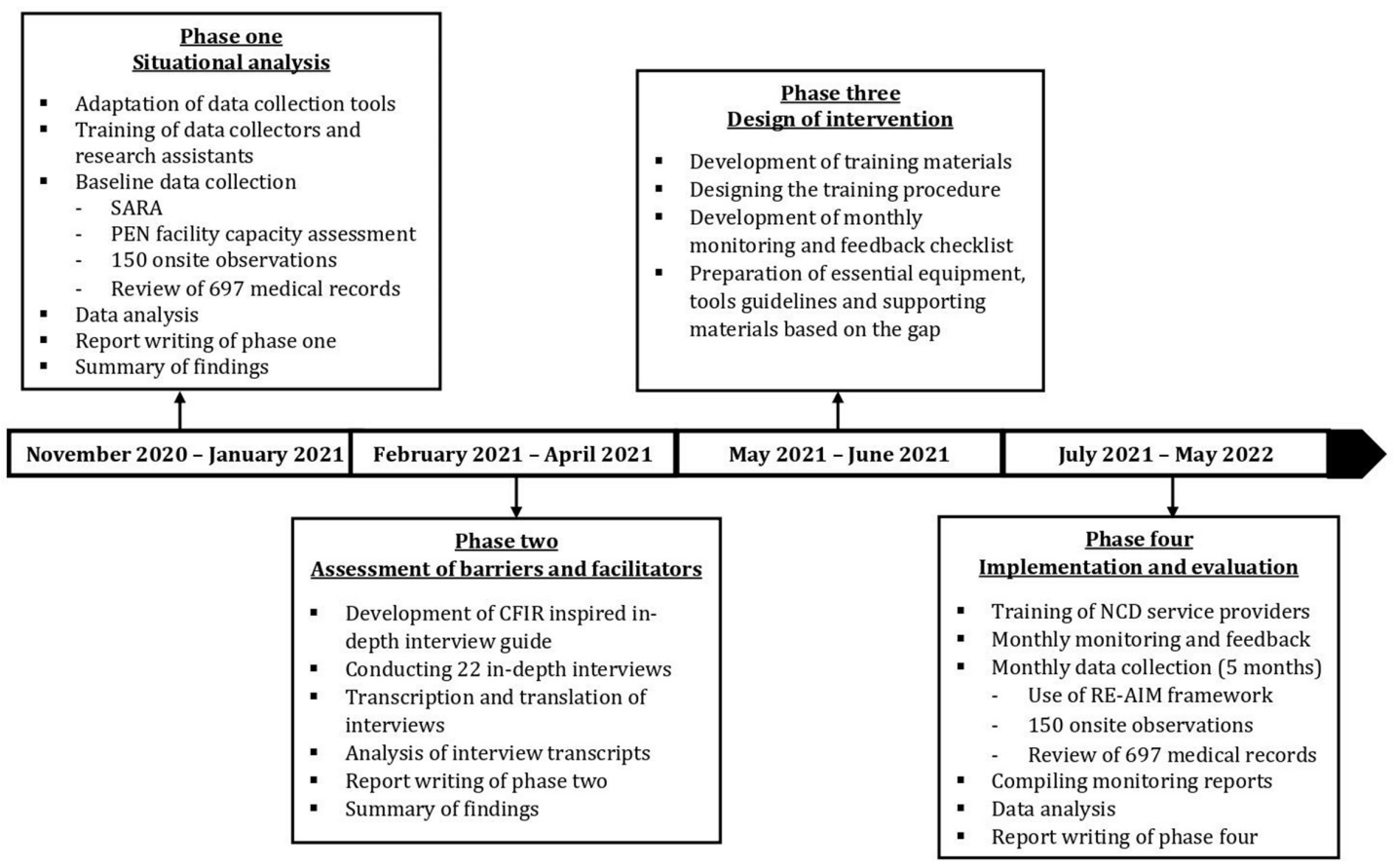

Figure 2 Schematic representation of the overall study design and timeline. CFIR, Consolidated Framework for Implementation Research; NCDs, non-communicable diseases; RE-AIM, reach, efficacy, adoption, implementation and maintenance; SARA, Service Availability and Readiness Assessment. 
2. Capacity of the selected 10 health centres to diagnose and manage NCDs will be conducted using the PEN facility capacity assessment ${ }^{4}$ and Service Availability and Readiness Assessment (SARA) ${ }^{18}$ tools. The tools include assessment of availability and functionality of essential diagnostic tools, medications, laboratory facilities and tests and human resources to provide NCD services.

3. Early detection and management of NCDs will be assessed using the following approaches by adapting the assessment tools from WHO PEN and the clinical and programmatic management of major NCDs, Ethiopia guideline. $^{1419}$

1. Onsite observation of patient healthcare provider interactions of patients $\geq 40$ years of age attending the OPD in the selected health centres will be conducted. The observation will be guided by an observation checklist. The checklist will include information about risk assessment, diagnosis and counselling on adherence to medication and dietary and lifestyle modifications. We will conduct three consecutive observations of patient healthcare provider interactions from each provider working in OPDs for patients $\geq 40$ years of age in the selected health centres resulting in a total of 15 observations from each health centre $(n=150)$ (figure 1). Data collection will be conducted on all days of the week until the desired sample size is achieved. Data will be collected at each health centre at the same time. Background information of healthcare providers including age, educational status, years of work experience and previous training on NCDs will be collected using a self-administered questionnaire after the observation.

2. Medical records of patients $\geq 40$ years of age during the baseline data collection will be reviewed to assess opportunistic screening, risk assessment, management of patients and extent of recording/documentation of information on assessments, medications, laboratory analysis and dietary and lifestyle characteristics of patients in the selected health centres. Patient medical records are paper based and stored in health centres. We estimated the number of patient's medical records to be reviewed using a single population proportion. The following assumption was considered to obtain the minimum sample size: the prevalence of hypertension among outpatient clients in Addis Ababa 34.7\%, ${ }^{20} 95 \%$ precision and adjusting for design effect of 2 , the calculation resulted in a minimum of 697 patient medical records to be reviewed. This sample will be proportionally allocated to the selected 10 health centres based on their monthly average caseload. Sampling frame will be obtained from each health centre triage and simple random sampling will be used to select patient medical records (figure 1).
Data collection procedure of phase one

The first phase of the study will be conducted from November 2020 to January 2021. Data on baseline situational analysis will be collected by 10 health professionals. The data collection process will be overseen by five research assistants who are also health professionals. Data collectors and research assistants will be trained on the data collection process and tools by the research team.

\section{Analysis of phase one}

Data from situational analysis, PEN facility capacity assessment, SARA, observation and review of medical records will be analysed using descriptive analysis techniques and will be presented using frequency tables, percentages, mean and median.

\section{Phase two: assessment of barriers and facilitators}

In this phase two, early detection and management of major NCDs will employ a qualitative study design. The research team will develop an in-depth qualitative interview guide using a consolidated framework for implementation research, which is compatible to analyse both patient-level and system-level determinants. The framework also accounts environmental thinking by providing theory-based constructs for developing context-specific models. ${ }^{21} 22$ The interview guide will be prepared in English, which will then be translated to the local language Amharic by a language expert and translated back to English to verify its consistency. The in-depth interview will target key stakeholders including healthcare providers working in OPD, pharmacy, laboratory, patients $\geq 40$ years of age (both patients with NCD and other conditions), health centre administrative, representatives of subcity, Addis Ababa Health Bureau, Ministry of Health, health centre HMIS and Pharmaceuticals Fund and Supply Agency (PFSA). PFSA is a sole government led supplier of medication, laboratory reagents and essential medical equipment in Ethiopia.

\section{Data collection procedure of phase two}

This phase of the study will be conducted from February 2021 to April 2021. We plan to conduct 20 in-depth interviews of key stakeholders. However, based on the saturation of the data, further theoretical sampling will be considered. Four experienced qualitative interviewers who are health professionals will conduct the in-depth interviews after receiving a training about the purpose of the study and components of the interview guide. All in-depth interviews will be audiotaped.

\section{Consolidated Framework for Implementation Research}

The aim of this study is to improve early detection and management of NCDs in primary healthcare services of Addis Ababa. It is important to understand the context of primary care facilities in order to document the nuances of the current scenario. Using theory-derived frameworks to improve the early detection and management of NCDs in low-income countries like Ethiopia will help to generate robust evidence to back up policies. Thus, we will use the 
Box 1 CFIR (Consolidated Framework for Implementation Research) domains and associated constructs

1. Intervention characteristics
Intervention source
Evidence strength and quality
Relative advantage
Adaptability
Trialability
Complexity
Design quality
20st
2. Outer setting
Patient needs and resources
- Cosmopolitanism
Peer pressure
3. External policy and incentives setting
- Structural characteristics
- Networks and communication
Culture implementation climate
4. Readiness for implementation
- Knaracteristics of individuals
- Enowledge and beliefs about the intervention
- Endividual stage of change
- Other personal attributes
Process

Consolidated Framework for Implementation Research (CFIR) framework to understand the contextual barriers and facilitators related to early detection and management of NCDs particularly hypertension and diabetes at primary healthcare level in Addis Ababa. ${ }^{21}$ Over the past years, the use of this framework is increasing, especially in helping healthcare systems identify why health interventions are not working and on identifying hurdles to and acceptability of new interventions. The framework is a synthesis of different implementation frameworks into one consolidated framework with 39 constructs organised under five domains (intervention characteristics, outer setting, inner setting, characteristics of individuals and process) (box 1). Our data collection tool will be designed to include all the five domains of the CFIR framework and we will try to cover as much constructs as possible depending on the saturation of the interviews.

\section{Data analysis of phase two}

Qualitative interviewers will transcribe the audiotaped in-depth interviews into Amharic and then translate it to English language. The authors of the study will verify the translated interviews against the audiotapes for accuracy. English version of the transcript will be prepared in text files and entered into Open Code software for processing and analysis. Two individuals with experience in qualitative analysis will make the coding line by line. We will follow thematic analysis based on the domains and constructs of CFIR framework. Codes, categories, patterns and quotations will be analysed into the preconceived codebook from the CFIR domains and constructs as they emerge in the interview transcripts. Accordingly, the barriers and the facilitators will be identified. Along with the finding from the quantitative study, the research team will triangulate the evidence to assist further planning and development of intervention protocol.

\section{Phase three: design of intervention protocol}

During this phase, we will design an intervention protocol tailored to address barriers and adaptation of the implementation on the existing systems to improve early detection and management of patients $\geq 40$ years of age. This design phase will be informed by the findings of the earlier phases and the HEARTS technical package and implementation guide. ${ }^{523}$ The intervention protocol will include the following capacity enhancement and monitoring components and will be developed from May 2021 to June 2021. The outlined intervention will be further strengthened based on the results of our baseline assessment and the identification of the barriers and facilitators.

1. Development of training materials including training module and job-aids guided by the findings of earlier phases and the WHO PEN and HEARTS packages. The training module will consist of information regarding early detection and management of NCDs, counselling skills, recording and reporting.

2. Training of healthcare providers working in OPD, laboratory pharmacy and triage, subcity mentors/supervisors, representatives from health centre administration, health centre HMIS representative, PFSA. The training will be provided by internists, endocrinologists and public health nutrition professionals from Addis Ababa University. A 3-day training will be provided once at the beginning of the implementation phase. It will include PowerPoint presentations, question and answer sessions and role-plays.

3. Provision of guidelines and supporting materials such as HEARTS technical package, job-aids and posters to be placed/posted inside the OPD, recording and reporting charts, copies of the national standard recording and reporting templates. Guidelines and supporting materials will be provided based on the gaps in the monthly monitoring reports throughout the implementation phase.

4. Provision and/or maintenance of essential equipment such as blood pressure and glucose measuring devices, weight scale, height measuring device/stadiometer and waist circumference measuring tape. Essential equipment will be provided and/or maintained based on the gaps in the monthly monitoring reports throughout the implementation phase. The study team will liaise with the Ministry of Health, Addis Ababa Health Bureau and non-governmental organisations to provide/maintain 
essential equipment, guidelines and supporting materials to deliver NCD services.

5. Facilitation of medication and laboratory reagent procurement from the national PFSA in case of stock out throughout the implementation phase. The study team will facilitate procurement by assisting the health centres in writing procurement request before a complete stock out to allow time for the procurement process. We will also assist health centres to have regular monitoring of the availability and functionality of essential medications, reagents and equipment.

6. Maintaining clean and comfortable environment in NCD service rooms for patient healthcare provider consultations. The study team will make sure that NCD service rooms are clean and comfortable during the monthly monitoring.

7. Monthly monitoring and feedback of the NCD services will be conducted for 5 months.

Phase four: implementation, monitoring and evaluation phase In this phase, we will implement the intervention protocol in the selected 10 health centres. After the training and provision of essential equipment, we will conduct monthly monitoring of the health centres with feedback for 5 months in addition to the regular monthly mentoring/supervision visits conducted by subcity mentors. We will adapt a checklist from WHO PEN package to guide the monitoring visits. The checklist will include availability and functionality of essential tools, medications, equipment and trained staff and regularity/functionality of the subcity mentorship and feedback programme. After each monitoring visit, a performance report will be prepared, and best practices/lessons learnt will be documented to capitalise the experience for the rest of the health centres. Appropriate written feedback will then be provided to respective health centres, NCD service providers and subcity mentors at each monitoring visit. The feedback will be provided by trained health professionals who are involved in the data collection process. We will also provide and/or maintain essential tools and equipment for health centres based on the gaps in the monitoring reports.

As part of continuous monitoring, we will assess early detection and management of NCDs every month for 5 months using the same observation checklist and patient medical records reviewing tool used during the baseline assessment. The variables in the observation checklist and patient medical record reviews will be organised using the RE-AIM framework by Glasgow et al $(1999)^{24}$ to evaluate certain dimensions of the intervention and implementation process (online supplemental table 1). The framework will assess five dimensions of the implementation at individual and organisation level; reach, efficacy, adoption, implementation and maintenance.

\section{Outcome measures}

This study will measure outcomes related to NCD detection and management at primary healthcare level.
Outcomes will be assessed through review of patient medical records, onsite observation of patient healthcare provider interactions, review of HMIS reports and monthly monitoring reports.

The outcomes to be measured in this study are as follows: Further, key outcome measures of the study under each dimensions of the RE-AIM framework are presented as a supplement along this paper (online supplemental table 1). We will display outcome measures for each data collection month from baseline to end line.

1. Assessment and counselling: $\%$ of individuals $\geq 40$ years of age for whom blood pressure, blood glucose and waist circumference measured, $\%$ of individuals $\geq 40$ years of age for whom cardiovascular risk estimated and $\%$ of individuals $\geq 40$ years of age who received dietary and lifestyle counselling in each month of data collection.

2. Treatment and control of NCDs: effectiveness of NCD management and control programme will be evaluated using indicators such as \% of individual who received antihypertensive and antidiabetic medications, $\%$ of individuals with blood pressure $>140 / 90 \mathrm{~mm} \mathrm{Hg}$, \% individuals with random blood sugar $>140 \mathrm{mg} / \mathrm{dL}$ or fasting blood sugar $>110 \mathrm{mg} /$ $\mathrm{dL}, \%$ individuals with cardiovascular risk $>30 \%, \%$ of individuals with high waist circumference $(>80 \mathrm{~cm}$ in women and $>90 \mathrm{~cm}$ in men), $\%$ individuals with hypertension and diabetes related complications and $\%$ individuals with lifestyle modifications in each month of data collection.

\section{Data collection procedure of phase four}

Phase four of the study will be conducted from July 2021 to May 2022. Trained health professionals involved during the baseline data collection and monitoring will collect data for this phase.

\section{Analysis of phase four}

Monthly monitoring reports will be compiled to produce a summary of the reports including best practices/lessons learnt and feedbacks. Data from observation checklists and review of medical records will be analysed using descriptive analysis techniques such as frequencies, percentages, mean and median. Monthly data for all collected variables will be presented in a table to show the changes/progresses each month. Trend of monthly reports will be compared using $\mathrm{X}^{2}$ for trend analysis.

\section{DISCUSSION}

A comprehensive understanding of the organisational situations, readiness, service availability and barriers and facilitators to early detection and management of NCDs are important to implement an innovative approach to integrate the service at the primary healthcare level. In addition, locally customised intervention towards NCDs 
will enhance the overall healthcare system and help policymakers to make evidence informed decisions.

In this study, a representative sample, one health centre from each subcity in Addis Ababa was selected. We excluded health centres with an ongoing intervention. Furthermore, the study aims to derive learning from context and develop a set of working interventions and best practices, which can be transferred to other settings. Thus, the findings from this study can be generalised to urban settings of Ethiopia and could serve as a lesson for other places with similar setting.

\section{ETHICS AND DISSEMINATION \\ Ethical considerations}

Ethical clearances were obtained from the Institutional Review Board (IRB) of the College of Health Sciences of the Addis Ababa University and Addis Ababa Health Bureau ethical review committee, with ethical clearance reference number 070/18/IM, dated 24 December 2018. Informed consent to participate in the study will be obtained from individual participants and selected health centres. All respondents will be provided the name, telephone number and email of the principal investigator and IRB contact details to contact if they have any questions about the study.

\section{Dissemination plan}

The main target audience for this study include Ministry of Health officials, policymakers, programme implementers, health workers, researchers and non-governmental organisations working to improve emerging health problems in low- and middle-income countries. Moreover, the finding from this study will help improve early detection of NCDs for populations at grass root level attending primary healthcare facilities. Any interested group can use this evidence to help improve early detection and management of NCDs health services.

\section{Patient and public involvement}

Patients or the public were not involved in the design, or conduct, or reporting, or dissemination plans of our research.

\section{Author affiliations}

${ }^{1}$ Department of Internal Medicine, Addis Ababa University College of Health Sciences, Addis Ababa, Ethiopia

${ }^{2}$ Department of Public Health Nutrition and Dietetics, Addis Ababa University College of Health Sciences, Addis Ababa, Ethiopia

${ }^{3}$ Centre for Innovative Drug Development and Therapeutic Trials for Africa (CDTAfrica), Addis Ababa University, Addis Ababa, Ethiopia

${ }^{4}$ School of Health Sciences, University of Brighton, Brighton, UK

${ }^{5}$ Department of Epidemiology and Biostatistics, Addis Ababa University College of Health Sciences, Addis Ababa, Ethiopia

Twitter Helen Yifter @HelenYifter, Abebaw Fekadu @abebawfekadu and Abraham Kebede @abrishya

Contributors HY, SG, ND, AF and THG conceived the project. HY, AO and AK designed development of situational analysis, intervention and implementation, monitoring and evaluation plan. All authors contributed to the refinement of situational analysis, intervention and implementation, monitoring and evaluation plan. $\mathrm{HY}, \mathrm{AO}$ and $\mathrm{AK}$ wrote the first draft of the manuscript. SG, ND, AF, THG and AM revised the manuscript draft. All authors contributed to the refinement of the study protocol and approved the final manuscript.

Funding The authors have not declared a specific grant for this research from any funding agency in the public, commercial or not-for-profit sectors.

Competing interests None declared.

Patient consent for publication Not required.

Provenance and peer review Not commissioned; externally peer reviewed.

Supplemental material This content has been supplied by the author(s). It has not been vetted by BMJ Publishing Group Limited (BMJ) and may not have been peer-reviewed. Any opinions or recommendations discussed are solely those of the author(s) and are not endorsed by BMJ. BMJ disclaims all liability and responsibility arising from any reliance placed on the content. Where the content includes any translated material, BMJ does not warrant the accuracy and reliability of the translations (including but not limited to local regulations, clinical guidelines, terminology, drug names and drug dosages), and is not responsible for any error and/or omissions arising from translation and adaptation or otherwise.

Open access This is an open access article distributed in accordance with the Creative Commons Attribution Non Commercial (CC BY-NC 4.0) license, which permits others to distribute, remix, adapt, build upon this work non-commercially, and license their derivative works on different terms, provided the original work is properly cited, appropriate credit is given, any changes made indicated, and the use is non-commercial. See: http://creativecommons.org/licenses/by-nc/4.0/.

\section{ORCID iDs}

Afrah Omer http://orcid.org/0000-0001-7775-4335

Abebaw Fekadu http://orcid.org/0000-0003-2219-0952

Abraham Kebede http://orcid.org/0000-0003-2551-502X

\section{REFERENCES}

1 Varghese C, Nongkynrih B, Onakpoya I, et al. Better health and wellbeing for billion more people: integrating non-communicable diseases in primary care. BMJ 2019;364:I327.

2 Narain JP. Integrating services for noncommunicable diseases prevention and control: use of primary health care approach. Indian J Community Med 2011;36:67-71.

3 Samb B, Desai N, Nishtar S, et al. Prevention and management of chronic disease: a litmus test for health-systems strengthening in low-income and middle-income countries. Lancet 2010;376:1785-97.

4 WHO. Implementation tools: package of essential noncommunicable (PEN) disease interventions for primary health care in low-resource settings. Geneva: World Health Organizaiton, 2013.

5 WHO. Hearts: technical package for cardiovascular disease management in primary health care., in WHO press. Geneva: World Health Organization, 2016.

6 NCD Alliances. Shaping the health systems of th efuture; case studies and recommendations for integrated NCD care. Switzerland: NCD Alliance Geneva, 2018.

7 Jakab M, Evetovits T, McDaid D. Health systems respond to noncommunicable diseases: time for ambition. Copenhagen, Demark: WHO Regional Office for Europe, 2018: 200-23.

8 Alwan A, Maclean DR, Riley LM, et al. Monitoring and surveillance of chronic non-communicable diseases: progress and capacity in highburden countries. Lancet 2010;376:1861-8.

9 WHO. Noncommunicable diseases country profiles 2018. Geneva: World Health Organization, 2018.

10 Bennett JE, Stevens GA, Mathers CD, et al. NCD countdown 2030: worldwide trends in non-communicable disease mortality and progress towards sustainable development goal target 3.4. Lancet 2018;392:1072-88.

11 WHO. The global burden of disease 2004 Update., W. press. Geneva: World Health Organization, 2008.

12 Tapela NM, Tshisimogo G, Shatera BP, et al. Integrating noncommunicable disease services into primary health care, Botswana. Bull World Health Organ 2019;97:142-53.

13 Checkley W, Ghannem H, Irazola V, et al. Management of NCD in low- and middle-income countries. Glob Heart 2014;9:431.

14 Federal Democratic Republic of Ethiopia Ministry of Health. Guidelines on clinical and programmatic management of major non communicable diseases. Addis Ababa, Ethiopia, 2016.

15 Federal Democratic Republic of Ethiopia Ministry of Health. Health and health-related indicators. Addis Ababa, Ethiopia: FDRE Ministry of Health, 2010. 
16 Ministry of Health. The Ethiopian health sector transformations plan II. Addis Ababa, 2015.

17 Curran GM, Bauer M, Mittman B, et al. Effectiveness-implementation hybrid designs. Med Care 2012;50:217-26.

18 WHO. Service availability and readiness assessment (SARA); implementation guide. Geneva: World Health Organization, 2015.

19 Martinez RE, Quintana R, Go JJ, et al. Surveillance for and issues relating to noncommunicable diseases post-Haiyan in region 8 . Western Pac Surveill Response J 2015;6:21-4.

20 Abebe S, Yallew WW. Prevalence of hypertension among adult outpatient clients in hospitals and its associated factors in Addis Ababa, Ethiopia: a hospital based cross-sectional study. BMC Res Notes 2019;12.

21 Warner G, Lawson B, Sampalli T, et al. Applying the consolidated framework for implementation research to identify barriers affecting implementation of an online frailty tool into primary health care: a qualitative study. BMC Health Serv Res 2018;18:395.

22 Safaeinili Net al. CFIR simplified: pragmatic application of and adaptations to the consolidated framework for implementation research (CFIR) for evaluation of a patient-centered care transformation within a learning health system. Learning Health Systems 2019;4.

23 WHO. HEARTS technical package for cardiovascular disease management in primary health care: implementation guide. Geneva: World Health Organizaiton, 2018.

24 Glasgow RE, Vogt TM, Boles SM. Evaluating the public health impact of health promotion interventions: the RE-AIM framework. Am J Public Health 1999;89:1322-7. 